\title{
Decitabine and Melphalan Fail to Reactivate p73 in p53 Deficient Myeloma Cells
}

\author{
Pierre-Samuel Gillardin ${ }^{1}$, Géraldine Descamps ${ }^{1}$, Sophie Maiga ${ }^{1}$, Benoit Tessoulin ${ }^{1}$, \\ Hanane Djamai ${ }^{1}$, Benedetta Lucani ${ }^{1}$, David Chiron ${ }^{1}$ (D), Philippe Moreau ${ }^{1,2}$, \\ Steven Le Gouill ${ }^{1,2}$, Martine Amiot ${ }^{1}$, Catherine Pellat-Deceunynck ${ }^{1, *}$ and \\ Agnès Moreau-Aubry ${ }^{1}$ \\ 1 CRCINA, INSERM, CNRS, Université d'Angers, Université de Nantes, 44007 Nantes, France; \\ pierre.gillardin@etu.univ-nantes.fr (P.-S.G.); geraldine.descamps@univ-nantes.fr (G.D.); \\ sophie.maiga@inserm.fr (S.M.); benoit.tessoulin@chu-nantes.fr (B.T.); djamai.hanane@gmail.com (H.D.); \\ benedetta.lucani@gmail.com (B.L.); david.chiron@inserm.fr (D.C.); philippe.moreau@chu-nantes.fr (P.M.); \\ steven.legouill@chu-nantes.fr (S.L.G.); martine.amiot@inserm.fr (M.A.); \\ agnes.aubry@univ-nantes.fr (A.M.-A.) \\ 2 Service d'Hématologie Clinique, Unité d'Investigation Clinique, CHU, 44093 Nantes, France \\ * Correspondence: catherine.pellat-deceunynck@univ-nantes.fr; Tel.: +33-228-080-298
}

Received: 25 November 2017; Accepted: 22 December 2017; Published: 23 December 2017

\begin{abstract}
Background: TP53 deficiency remains a major adverse event in Multiple Myeloma (MM) despite therapeutic progresses. As it is not possible to target TP53 deficiency with pharmacological agents, we explored the possibility of activating another p53 family member, p73, which has not been well studied in myeloma. (2) Methods: Using human myeloma cell lines (HMCLs) with normal or abnormal TP53 status, we assessed TP73 methylation and expression. (3) Results: Using microarray data, we reported that TP73 is weakly expressed in 47 HMCLs and mostly in TP53 wild type (TP53 ${ }^{\text {wt }}$ ) HMCLs $(p=0.0029)$. Q-RT-PCR assays showed that TP73 was expressed in $57 \%$ of TP53 ${ }^{w t}$ HMCLs (4 out of 7 ) and $11 \%$ of TP53 abnormal (TP53 $\left.{ }^{a b n}\right)$ HMCLs ( 2 out of 18) $(p=0.0463)$. We showed that TP73 is silenced by methylation in TP53 ${ }^{a b n}$ HMCLs and that decitabine increased its expression, which, however, remained insufficient for significant protein expression. Alkylating drugs increased expression of TP73 only in TP5 $3^{\text {wt }}$ HMCLs but failed to synergize with decitabine in TP53 $3^{\text {abn }}$ HMCLs. (4) Conclusions: Decitabine and melphalan does not appear as a promising combination for inducing p73 and bypassing p53 deficiency in myeloma cells.
\end{abstract}

Keywords: multiple myeloma; p53; p73; CpG methylation; decitabine; alkylating drugs

\section{Introduction}

Multiple Myeloma (MM) is characterized by an important biological heterogeneity related to recurrent chromosomal abnormalities, which occurs early in the disease development at the MGUS (Monoclonal Gammopathy of Undetermined Significance) stage [1]. The recurrent chromosomal abnormalities are $\operatorname{IgH}$ gene translocation with recurrent partners located on chromosomes 4, 6, 11, 16 and 20 or hyperdiploidy of odd chromosomes [1]. Different prognoses and overall survivals are associated with this first heterogeneity. However, and independently from this heterogeneity, the most adverse prognosis is related to chromosomal deletion of TP53 [2]. Mutations of TP53 were exclusively found in myeloma cells displaying a hemi-deletion of the short arm of chromosome 17 (del17p) [3]. The frequency of TP53 mutations, which is low at diagnosis (around 3\%), increases with relapses and is high in plasma cell leukemia (PCL, $>30 \%$ ) and human myeloma cell lines (HMCLs, $>70 \%$ ), these latter mostly deriving from extramedullary MM and mainly from PCL [4-8]. Loss of function of mutant p53 is believed to be related to conformation deficiency: therefore, small molecules, such as 
RITA and Prima- $1^{\text {Met }}$, which were selected for their ability to induce cell death in p53 mutated cells were shown to bind to p53 protein [9]. However, in our hands, both molecules induced massive cell death but their efficacy was unrelated to TP53 status and p53 expression [10,11]. Because del(17)p and TP53 mutations, which are irreversible, are an adverse event whatever the treatment regimen, alternative treatments that bypass or circumvent p53 are needed. For instance, the p53 family member p73 could be of interest as p73 is also able to transactivate pro-apoptotic genes [12,13]. Moreover, TP73 is rarely mutated but frequently silenced by $\mathrm{CpG}$ methylation in hematological malignancies and in multiple myeloma (MM) [14-17]. TP73 expression might thus be reactivated by drugs preventing CpG methylation after DNA replication such as decitabine. To assess the interest in activating p73 in myeloma cells, we studied p73 expression and regulation, TP73 methylation and TP73 sequencing in a large panel of human myeloma cell lines HMCLs with a normal or abnormal TP53 status [7,18].

\section{Results}

\subsection{TP73 Is Preferentially Constitutively Expressed in TP53 ${ }^{\text {wt }}$ HMCLs}

We assessed expression of TP73 mRNA in 47 HMCLs using Affymetrix microarray data [7]. Although expression level was very low, HMCLs with normal TP53 status (TP53 ${ }^{\text {wt }}$ ) appeared to significantly express higher level of TP73 mRNA than TP53 ${ }^{a b n}$ HMCLs $(p=0.0029$, Mann-Whitney test, Figure 1). To confirm this observation, we further selected 25 HMCLs displaying either a normal $(n=7)$ or an abnormal TP53 status $\left(n=18,3\right.$ TP53 deleted $\left(\right.$ TP53 $\left.{ }^{\text {del }}\right)$ and 15 TP53 mutated (TP53 $\left.{ }^{\text {mut }}\right)$ HMCLs) (Table 1) $[7,11,18]$. Status of TP53 and TP73 was determined by sequencing of RT-PCR products and/or whole exon sequencing. Q-RT-PCR assays showed that TP73 was expressed in four out of seven TP53 HMCLs (AMO1, MDN, MM1S, NAN11), in one out of three TP53 ${ }^{\text {del }}$ HMCLs (JJN3) and in one out of fifteen TP53 ${ }^{\text {mut }}$ HMCLs (XG11), $p=0.0463$, Mann-Whitney test, (Figure 1B). p73 expression was confirmed at the protein level using Western blotting for MDN, MM1S, NAN11, JJN3 and XG11 but not for AMO1 (Figure 1C). Whole exon sequencing showed that only KMM1 and XG11 displayed a missense mutation (A211S and I626V, respectively). However, XG11, but not KM11, expressed TP73 and p73. The strong p73 expression in XG11 might be related to the presence of the mutation within the transactivation inhibitory domain, which was reported to be involved in p73 degradation [19].

Table 1. Human myeloma cell lines (HMCL) characteristics.

\begin{tabular}{|c|c|c|c|c|c|c|}
\hline \multicolumn{2}{|c|}{ HMCL } & \multicolumn{2}{|c|}{ TP53 Status and Expression } & \multicolumn{3}{|c|}{ TP73 Status and Expression } \\
\hline Name & Translocation & TP53 & p53 & TP73 & Q-PCR & p73 \\
\hline AMO1 & unknown & wt & + & wt & + & - \\
\hline MDN & $(11 ; 14)$ & wt & + & wt & + & + \\
\hline MM1S & $(14 ; 16)$ & wt & + & wt & + & + \\
\hline NAN9 & $(4 ; 14)$ & wt & + & wt & - & - \\
\hline NAN11 & $(14 ; 16)$ & wt & + & wt & + & + \\
\hline XG6 & $(16 ; 22)$ & wt & + & wt & $+1-$ & - \\
\hline XG7 & $(4 ; 14)$ & wt & + & wt & $+1-$ & nd \\
\hline JJN3 & $(14 ; 16)$ & deletion & - & wt & + & + \\
\hline KMS11 & $(4 ; 14)$ & deletion & - & wt & - & - \\
\hline NAN8 & $(4 ; 14)$ & disrupted $^{1}$ & - & wt & - & - \\
\hline JIM3 & $(4 ; 14)$ & R273C & + & wt & - & - \\
\hline Karpas620 & $(11 ; 14)$ & $\mathrm{C} 135 \mathrm{Y}$ & + & wt & - & nd \\
\hline KMM1 & $(6 ; 14)$ & $\mathrm{C} 135 \mathrm{~F}$ & + & A211S & - & nd \\
\hline KMS12PE & $(11 ; 14)$ & R337L & + & wt & - & - \\
\hline LP1 & $(4 ; 14)$ & E286K & + & wt & - & - \\
\hline L363 & $(20 ; 22)$ & $\mathrm{S} 261 \mathrm{~T}^{2}$ & - & wt & - & - \\
\hline NAN1 & $(14 ; 16)$ & E180STOP & - & wt & - & nd \\
\hline NAN6 & $(14 ; 16)$ & Indel $^{3}$ & +5 & wt & - & nd \\
\hline NAN7 & $(11 ; 14)$ & Indel ${ }^{4}$ & - & wt & - & nd \\
\hline OPM2 & $(4 ; 14)$ & $\mathrm{R} 175 \mathrm{H}$ & + & wt & - & - \\
\hline SKMM2 & $(11 ; 14)$ & $\mathrm{K} 132 \mathrm{~N}$ & + & wt & - & nd \\
\hline XG11 & $(11 ; 14)$ & C135Y & + & $\mathrm{I} 626 \mathrm{~V}$ & + & + \\
\hline XG1 & $(11 ; 14)$ & $\mathrm{Y} 126 \mathrm{~N}$ & + & wt & $+1-$ & nd \\
\hline$X G 2$ & unknown & C176Y & + & wt & $+1-$ & nd \\
\hline XG5 & $(11 ; 14)$ & $\mathrm{R} 282 \mathrm{~W}$ & + & wt & - & - \\
\hline
\end{tabular}

nd: not done; ${ }^{1}$ disrupted by amplification of exons $1,2,3,4,5$ and $6 ;^{2}$ lack of intron 7 splicing; ${ }^{3}$ deletion of exons 7 , 8 and $9 ;{ }^{4}$ deletion of exon $11 ;{ }^{5}$ truncated form. 


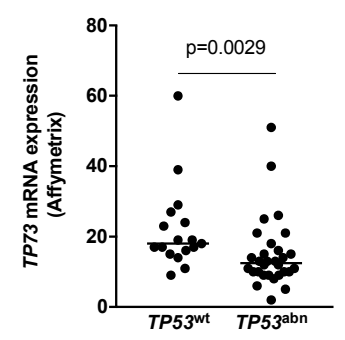

B
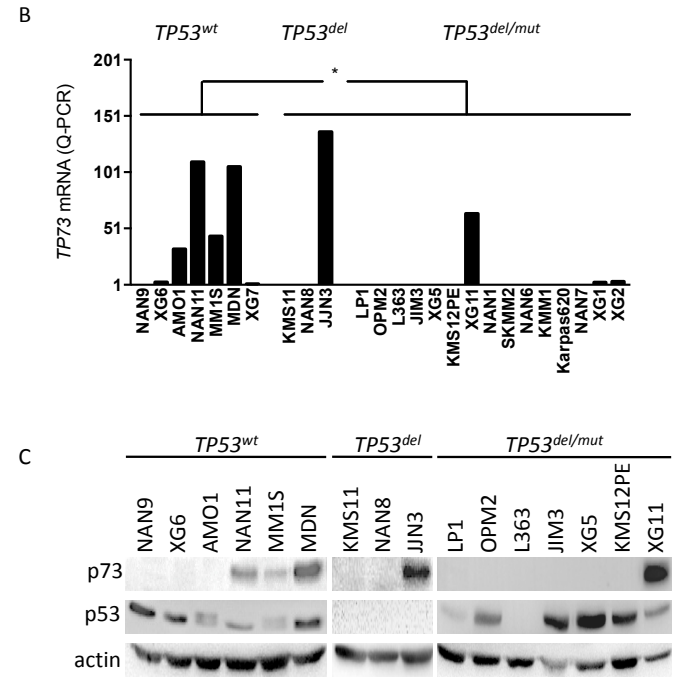

Figure 1. Constitutive TP73 expression was mainly restricted to TP53 ${ }^{\text {wt }}$ HMCLs: (A) Constitutive expression of TP73 in 47 HMCLs was performed by microarray (Affymetrix) and analyzed according to TP53 status. (B) Constitutive expression of TP73 was mainly restricted to TP53 ${ }^{w t}$ HMCLs. Expression was assessed by Q-PCR in 25 HMCLs. The histograms represent the mean \pm SEM of 3 experiments (SEM fall within the symbols). ${ }^{*}, p<0.05$. (C) Constitutive expression of p73 was mainly restricted to $T P 73^{+}$HMCLs. Expression of p73 and p53 was assessed by Western blotting with anti-p73 and anti-p53 antibodies.

\subsection{Decitabine Decreased TP73 Methylation and Induced TP73 Expression}

To assess the methylation level in TP73 promoter, we performed nested methyl-specific PCR (MS-PCR) targeting the CpG island upstream of the gene (Figure 2). PCR-1 was run on bisulfite converted DNA with primers that did not involve CpG. The PCR-1 product was then used for three nested PCRs; two, respectively, specific of unconverted CpG (methylated PCR, M-PCR) and converted CPG (unmethylated PCR, U-PCR) sequence; and one quantifying the PCR-1 product (PCR-Q, Figure 2). PCRs were run in HMCLs treated or not with decitabine to assess the TP73 methylation status (Figure 3A). In parallel, we measured the expression level of TP73 by Q-PCR (Figure 3B). In JIM3, KMS12PE and XG5, TP73 was found methylated and decitabine induced a decrease in M-PCR and an increase in U-PCR, which correlated with an increase in TP73 expression. However, despite the increase in TP73 expression, we could not detect any protein. Because anti-p73 Ab was directed against the $\mathrm{N}$ terminal part of the protein (amino acids 1-62) and could thus not detect $\Delta \mathrm{Np} 73$, we performed three RT-PCRs to assess the total TP73 expression (exons 7-10), the full-length TAp73 (exons 2-6) and the $\triangle N p 73$ (exons $3^{\prime}-6$ ) isoforms, respectively. The TAp73 isoform was increased in the three $T P 53^{m u t}$ HMCLs, although in KMS12PE the $\triangle N p 73$ isoform was the main increased isoform (Figure $3 C$ ). The data suggested that the lack of p73 detection by Western blotting was not related to the lack of TAp73 transactivation, but rather to a weak transactivation. 
GCCGGCGCCTCTAGGGCCCCCCAGATCGCGCAGACCCTGACATCCCGCCTGGCCCTGGGTTCTGGGAGCTGAGAGCCGGCCAGGGTCCTGCTCGTACC $+:++|++::|:|||||::::::||||++++||||:::|||:||:::++:||||:::||||||:||||||:||||||:++|::|||||::||:|++| \mid:$ : GTCGGCGTTTTTAGGGTTTTTTAGATCGCGTAGATTTTGATATTTTCGTTTGGTTTTGGGTTTTGGGAGTTGAGAGTCGGTTAGGGTTTTGTTCGTAT TCCGGGCGCCCAGCCTCGGGTCTGCTCCCCGCGGACGCCCCAACCTCCCCGGCCGAATGGATGGTGGTGCGCGCGCGTCCTACTCCGGCGGTGCCGGCC $|:++||++:::||::|++|||:||:|:::++++||++::::||::|:::++|:++|||||||||||||||++++++++|::||:|:++|++|||:++|::$
TTCGGGCGTTTAGTTTCGGGTTTGTTTTTCGCGGACGTTTTAATTTTTTCGGTCGAATGGATGGTGGTGCGCGGCGTTTTATTTCGGCGGTGTCGGTT

TTTTCTGTTGCCAAAACTAGACCCAAACCTCTGCATGGGATTCGTCTTTGGGTCCCCACCCCGTGCGCCCAGCAAACAGTGGGTGAGCCATGAAGATGT

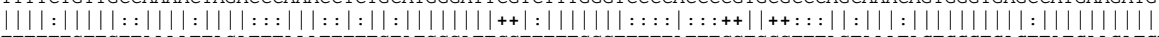
TTTTTGTTGTTAAAATAGATTTAAATTTTGTATGGGATTCGTTTTTGGGTTTTTATTTCGTGCGTTTAGTAAATAGTGGGTGAGTTATGAAGATGT GCGAGTCAGCCGGACCCTCCCCGTCAGGCGCGGACCCGCTGCGGCCAGAGAACCCAGTCTGCGCCAGCCCGGCTCGCTCGCGAAGCCACGGGCTTCACT

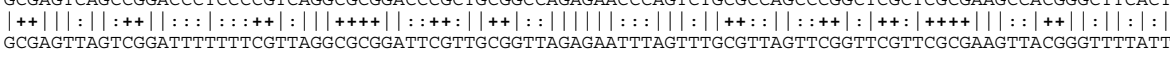

GACG CGACTTTCCAAGACGTGGGGGTCACCATGGGCAGAGGACATCGGTTCGGAGCCAGATCACGGGCCCCATAAGCATCAGACCATAAGCAGCGCCG

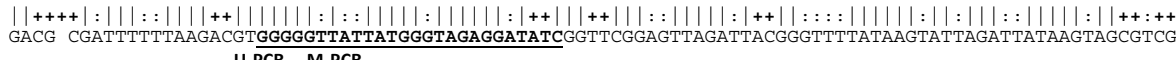

CCACTGAGAGCCGCTCGGAACTCGCCCAGCATGTCGGGTCCCCTAGCCAGGGCCTGGTGTACGTGGTCGAGGGCCCTGGAAGCCCCGATGGCCTAGGAG

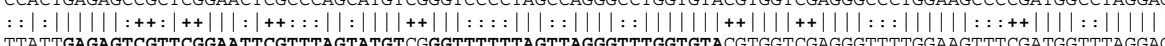
U-PCR M-PCR

A

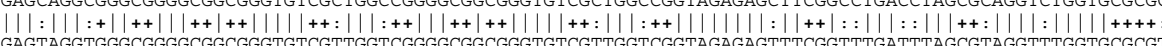

AGAGAACAACTCCAAGCGCACCGACGCCCGCGAGCTCCTTCCAAACACCGAACGGGATCCAGAGCCCGAGCCCACAGGCGGCGGCCGGGGGAGGGAGCA ||||||$|:||:|::|||++:|:++|++::++++||:|::||::|||:|:++||++||||::||||::++||:::|:|||++|++|:++||||||||||: \mid$

GGGTGCTGGCCGCCGCCCGGGAGTGTTCGCGTCCTGGGTGACCCCTGGAAGGACGTGGGGCCCAAACTCCGGCTGGGGTTGGGAGAGCAGCCCCCAGAG ||||||$:|||:++:++::++||||||||+++||:||||||||:::=||||||||++|||||:=:|||:|:++|:||||||||||||||||:||:::::|||||$

GCTCTCCGCGGGATCCTCTGCCGGGCGGGACCGTGGCTCCACAGGAGAAGTGGGTGGCAAGCCCTGCTTTGGCGGAAAGCAGCCGTTCCCCTCCTCCTGC $|:|:|:++++||||::|:|||:++||+|||:++|||:|:::|:||||||||||||||||:||:::|||:|||||++|||||:|||:++||::::||::|::||||$ GTTTTTGCGGGATTTTTTGTCGGGCGGGATCGTGGTTTTATAGGAGAAGTGGGTGGTAAGTTTTGTTTGGCGGAAAGTAGTCGTTTTTTTTTTTTTG PCR-1

GCCTGGGGCGGCGCCCCTCACCCCTGTTCCCCGCCCCTCACCCCTGTTCCCCGCCGGCCACATCCCCTGCCCCTTGGATTCCAAGCGCCCCGCGCGCCG

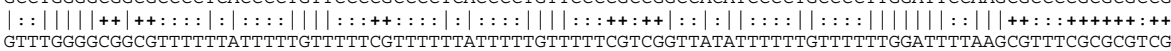

AGGAGCCCAGCGCTAGTGGCGGCGGCCAGGAGAGACCCGGGTGTCAGGAAAGATGGGCCGTCTGGGGGACAGCAGGGAGTCCGGGGGAAACGCAGGCGT

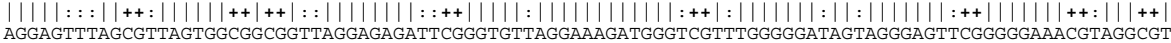

CGGGCACAGAGTCGGCACCGGCGTCCCCAGCTCTGCCGAAGATCGCGGTCGGGTCTGGCCCGCGGGAGGGGCCCTGGCGCCGGACCTGCTTCGGCCCTG

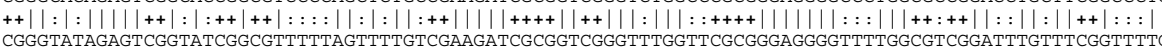

CGGGTATAGAGTCGGTATCGGCGTTITAGTTTTGTCGAAGATCGCGGTCGGGTTGGTCGCGGGAGGGGTTTGGCGTCGGATTMTTTCGTTTT ++||||$++|::|++:++||:|:||:||||||++|++++++::||||||++|++|||||||||++|||:||||++++::++||||::=++|:||||||++++\mid$

CCAGCTGGAGAGGCGGAGCGCCGGGAGGAGACCTTGGCCCCGCCGCGACTCGGTGGCCCGCGCTGCCTTCCCGCGCGCCGGGCTAAAAAGGCGCTAACG

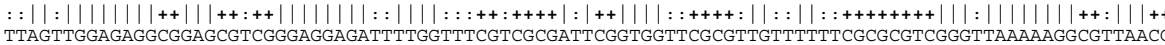

CCCGCGGCCGCCTACTCCCCGCGGCGCCTCCCCTCCCCGCGCCCATATAACCCGCCTAGGGGCCGGGCAGCCCGCCCIGCCTCCCCG

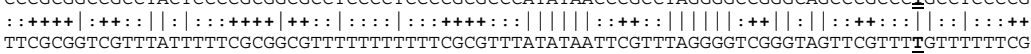

$+1$

Figure 2. Targeting of $\mathrm{CpG}$ islands in TP73. Upper and lower sequences represent the native and bisulfite converted TP73 DNA sequence, respectively. The CpG are indicated with "+". Primers used for PCR1, M-PCR (Methylated-PCR), U-PCR (Unmethylated methylation) and PCR-Q (Quantifying PCR) are indicated by underlines. +1 indicates the first transcripted nucleotide. 


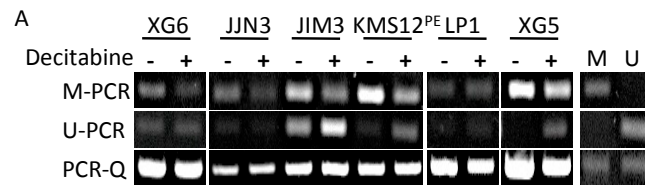

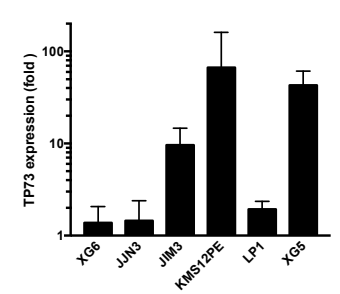

C

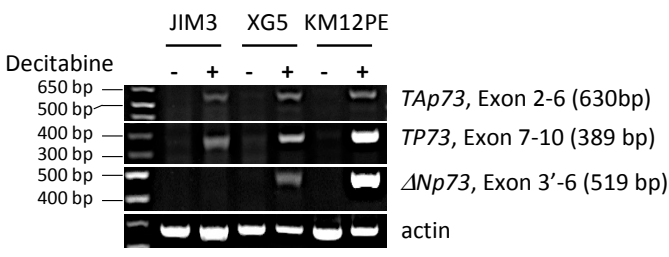

D

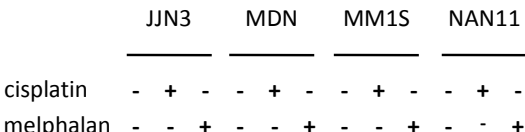

melphalan - - + - + + - + + +

$500 \mathrm{bp}$ 二

$500 \mathrm{bp}=$

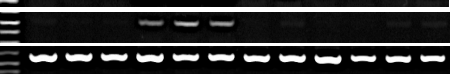

$\Delta N p 73$, Exon 3'-6

actin

E
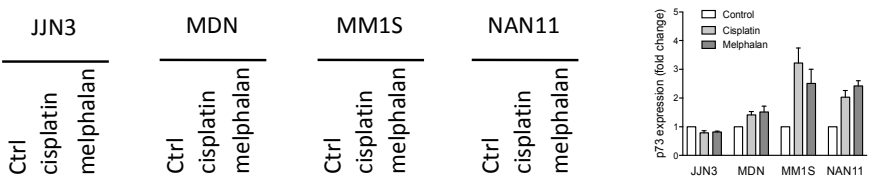

p73
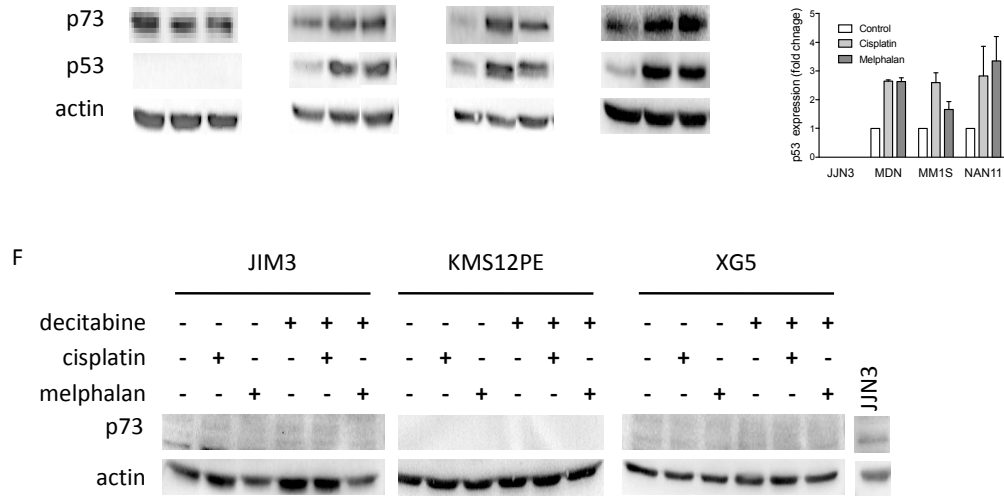

Figure 3. Cisplatin or melphalan induced the expression of TAp73 isoform in TP53 ${ }^{\text {wt }}$ HMCLs but not in TP53 ${ }^{a b n}$ HMCLs. (A) TP73 is mainly methylated in TP53 ${ }^{m u t}$ HMCLs. MS-PCRs were performed after bisulfite conversion of DNA extracted from HMCLs treated or not with decitabine ( $5 \mu \mathrm{M}, 72 \mathrm{~h})$. M and U indicated methylated and unmethylated control DNAs. (B) Decitabine induced TP73 expression. TP73 expression was assessed by Q-PCR, as described in the legend of Figure 1. (C) Decitabine induced expression of several TP73 isoforms. RT-PCRs were run on cDNAs from control or decitabine-treated HMCLs $(5 \mu \mathrm{M}, 72 \mathrm{~h})$, as indicated in the figure. (D) Cisplatin or melphalan increased the expression of TAp73 isoform. RT-PCR expression of TAp73 and $\triangle N p 73$ was performed after a 16-h treatment with melphalan or cisplatin. TP53 ${ }^{\text {wt }}$ HMCLs were treated with $7 \mu \mathrm{M}$ of melphalan or cisplatin, JJN3 cells were treated with $30 \mu \mathrm{M}$ of melphalan or $20 \mu \mathrm{M}$ of cisplatin. (E) Cisplatin or melphalan increased the expression p73 in TP53 ${ }^{w t}$ HMCLs. Western blots were performed after an overnight treatment with melphalan or cisplatin as described in (A). Right part represents the quantification of p73 and p53 over actin expression from two independent experiments (mean \pm SEM). (F) Decitabine and melphalan or cisplatin failed to induce p73 expression in TP53 ${ }^{\text {mut }}$ HMCLs. HMCLs were treated $72 \mathrm{~h}$ with decitabine $(5 \mu \mathrm{M})$ prior to an overnight treatment with $45 \mu \mathrm{M}$ of melphalan or $55 \mu \mathrm{M}$ of cisplatin. JJN3 proteins were loaded as a control of p73 expression. 


\subsection{Melphalan and Cisplatin Induced TP73 Expression in TP53 ${ }^{\text {wt }}$ HMCLs but Not in Decitabine-Treated TP53 ${ }^{\text {mut }}$ HMCLs}

To further study TP73 regulation, we treated four HMCLs that constitutively expressed p73 with alkylating drugs, melphalan and cisplatin and determined by RT-PCR and Western blotting the regulation of TP73 and p73. Regulation of p53 expression was assessed as a control of drug response [20]. Alkylating drugs increased the expression of the p73 full-length isoform in the TP53 $3^{\text {wt }}$ MDN, MM1S and NAN11 HMCLs but not in TP53 deleted (TP53 ${ }^{\text {del }}$ ) JJN3 HMCL (Figure 3D). MDN also expressed the $\Delta N p 73$ isoform that was slightly increased by the drugs. By Western blotting, we confirmed that melphalan and cisplatin similarly increased p73 expression in the three TP53 ${ }^{\text {wt }}$ HMCLs but failed to increase p73 expression in TP53 ${ }^{\text {del }} \mathrm{JJN} 3$ (Figure 3E). As expected, p53 expression was increased by both drugs in the TP53 ${ }^{w t}$ MDN, MM1S and NAN11 HMCLs but not in TP53 ${ }^{d e l}$ JJN3 cells [20].

We then assessed whether melphalan could increase expression of p73 in decitabine-treated TP53 ${ }^{m u t}$ HMCLs. We selected the three HMCLs that displayed a strong increase in TP73 expression upon decitabin treatment, i.e., JIM3, KMS12PE and XG5 (Figure 3B). As shown in Figure 3F, alkylating drugs failed to increase any p73 expression that remained undetectable in the 3 TP53 ${ }^{\text {mut }}$ HMCLs.

\section{Discussion}

In myeloma, regulation of p73 expression has not been deeply investigated yet. For instance, TP73 expression has been shown to be induced by PRIMA-1 ${ }^{\text {Met }}$ in several HMCLs including JJN3 in which p73 was reported to be partly involved in PRIMA- $1^{\text {Met }}$-induced cell death $[17,21]$. However, we did not confirm these results in JJN3, suggesting that JJN3 might be misidentified between the different laboratories. In hematological malignancies, TP73 that does not display frequent mutations is known to be silenced by $\mathrm{CpG}$ methylation [14]. Indeed, whole exon sequencing showed that only two HMCLs (KMM1 and XG11) harbored a mutation in TP73 gene. We thus assessed TP73 methylation and regulation in a large number of HMCLs. Our results showed that TP73 is mainly silenced by methylation and that the methylation inhibitor decitabine reversed the methylation-mediated silencing. However, the TP73 expression remained too weak for allowing a detectable p73 expression by Western blotting. We showed that decitabine-induced expression of both $T A p 73$ and $\triangle N p 73$ isoforms: $\Delta N p 73$, which is lacking the transactivation domain, is known to inhibit TAp73 binding to DNA [19]. In KMS12PE, the high decitabine-induced expression of $\triangle N p 73$ isoform might explain the lack of $p 73$ detection by Western blotting because the anti-p73 Abs was directed against the $\mathrm{N}$ terminal part of the protein (aa 1-62). TP73 gene also harbors an intrinsic CpG island that governs $\triangle N p 73$ isoform transcription. Thus, TP73 gene appears particularly methylated in MM cells and demethylation might equally induce expression of $T A p 73$ and $\triangle N p 73$ isoforms, the latter being an inhibitory isoform [19]. We found that p73 was constitutively expressed in six HMCLs: MDN, MM1S, NAN11 and XG10 expressed a wildtype p53 protein, JJN3 lacked p53 expression and XG11 expressed a mutant p53 protein. Of note, p73 was overexpressed in XG11 despite a moderate TP73 expression suggesting a stabilization/lack of degradation of the protein that might be related to the presence of the mutation in the transactivation inhibitory domain, which is involved in degradation [22]. In three out of four HMCLs that constitutively expressed p73 and displayed an unmethylated profile of CpG, alkylating drugs increased both TP73 and p73 expression: this regulation seemed to be restricted to TP53 HMCLs as it did not occur in the TP5 ${ }^{\text {del }} \mathrm{JJN} 3 \mathrm{HMCL}$. This finding is consistent with the possible regulation of TP73 expression via p53/p21/E2F1, which can only occur in TP53 ${ }^{\text {wt }}$ HMCLs [23]. Indeed, alkylating drugs were unable to increase 773 expression in the three TP53 ${ }^{\text {mut }}$ HMCLs that displayed a TP73 expression after decitabine treatment. Moreover, MDM2, which stabilizes p73 instead of inducing its degradation [24], could take a part in the p73 increase induced by alkylating drugs, because MDM2 is a p53 target gene mostly expressed in TP53 ${ }^{\text {wt }}$ HMCLs [18]. Nevertheless, the role of p53 in melphalan-induced p73 regulation, if any, requires further investigations. On the other hand, TP73 expression was shown to be regulated by HDAC: indeed, the HDAC inhibitor sodium butyrate 
increased the "free" E2F1 pool and therefore TP73 transactivation independently from p53 [25]. We did not investigate the HDAC-mediated regulation of p73 in myeloma cells but it would be of interest to study HDAC inhibitors in combination with decitabine in p53 deficient cells. In summary, our data show that decitabine and melphalan does not appear as a promising combination for bypassing p53 deficiency in myeloma cells.

\section{Materials and Methods}

\subsection{HMCLs and Reagents}

HMCLs were previously described [7,11]. Unmethylated/methylated control DNAs, and the bisulfite conversion kit were purchased from Active Motif (La Hulpe, Belgium). Anti-p73 A300-126A and anti-p53 DO-1 Abs were purchased from Bethyl Laboratories (Euromedex, Souffelweyersheim, France) and Millipore (Saint-Quentin en Yvelines, France), respectively. Anti-actin was purchased from Millipore. Cisplatin and melphalan were purchased from Sigma Aldrich (Saint-Quentin-Fallavier, France). Quantitative PCR probes were purchased from Taqman (Thermofisher, Saint-Herblain, France). Quantitative RT-PCR assays were performed as previously described [7].

\subsection{Bisulfite Treatment, Methylation Specific PCRs and RT-PCR}

DNAs were treated with bisulfite as indicated by the supplier. Methylation specific PCRs (M-PCR, U-PCR) and quantifying PCR (PCR-Q) were performed after a first amplification (PCR1). PCR1, M-PCR, U-PCR and PCR Q were run with 25, 17, 20 and 19 cycles, respectively. The following primers were used: PCR-1 GTTTTGGGTTTTGGGAGTTGAGAG and ACCACCCACTTCTCCTATAAAA (874 bp; M-PCR GGGGTTATTATGGGTAGAGGATATC and ACATACTAAACGAATTCCGAACGACTC (109 bp); U-PCR GGGTTATTATGGGTAGAGGATATT and ACATACTAAACAAATTCCAAACAACTCTC $(112 \quad$ bp $) ; \quad$ and $\quad$ PCR $Q$ TAAATAGTGGGTGAGTTATGAAGATGT and TACACCAAACCCTAACTAAAAAACC (285 bp). Extracted RNAs were reversed transcripted and amplified as previously described [12].

For TP73 RT-PCR assays, the following primers were used: TAp73 ex2-6 CACCACGTTTGAGCACCTCT and AGATTATTGCCTTCCACGCG (630 bp); TP73 ex7-10 GACGGAATTCACCACCATCCT and CCAGGCTCTCTTTCAGCTTC (389 bp); and $\Delta N p 73$ ex3'-6 CCATGCTGTACGTCGGTGAC and CCAAATCCTTCTCCCTATCC (519 bp). For TP73 Q-PCR assays, the TP73 (Hs01056230_m1) and RPL37A (Hs01102345_m1) probes were used.

\subsection{Western Blotting}

Expression of p53 and p73 was determined by Western blotting as previously described [11]. A minimum of $80 \mu \mathrm{g}$ of proteins was loaded in each lane.

Acknowledgments: The work was supported by a grant from Ligue Grand-Ouest Contre Le Cancer and by Actions Cancer 44.

Author Contributions: David Chiron, Philippe Moreau, Steven Le Gouill, Martine Amiot, Catherine Pellat-Deceunynck and Agnès Moreau-Aubry designed research; Pierre-Samuel Gillardin, Géraldine Descamps, Sophie Maiga, Benoit Tessoulin, Hanane Djamai and Benedetta Lucani acquired data; Pierre-Samuel Gillardin, Catherine Pellat-Deceunynck and Agnès Moreau-Aubry analyzed data; and Catherine Pellat-Deceunynck and Agnès Moreau-Aubry wrote the paper. All authors approved the final version of the paper.

Conflicts of Interest: The authors declare no conflict of interest.

\section{Abbreviations}

HMCL Human Myeloma Cell Line

MM Multiple Myeloma

MS-PCR Methylation-specific PCR 


\section{References}

1. Fonseca, R.; Bergsagel, P.L.; Drach, J.; Shaughnessy, J.; Gutierrez, N.; Stewart, A.K.; Morgan, G.; van Ness, B.; Chesi, M.; Minvielle, S.; et al. International Myeloma Working, International Myeloma Working Group molecular classification of multiple myeloma: Spotlight review. Leukemia 2009, 23, 2210-2221. [CrossRef] [PubMed]

2. Avet-Loiseau, H.; Attal, M.; Campion, L.; Caillot, D.; Hulin, C.; Marit, G.; Stoppa, A.M.; Voillat, L.; Wetterwald, M.; Pegourie, B.; et al. Long-term analysis of the IFM 99 trials for myeloma: Cytogenetic abnormalities [t(4;14), del(17p), 1q gains] play a major role in defining long-term survival. J. Clin. Oncol. 2012, 30, 1949-1952. [CrossRef] [PubMed]

3. Lode, L.; Eveillard, M.; Trichet, V.; Soussi, T.; Wuilleme, S.; Richebourg, S.; Magrangeas, F.; Ifrah, N.; Campion, L.; Traulle, C.; et al. Mutations in TP53 are exclusively associated with del(17p) in multiple myeloma. Haematologica 2010, 95, 1973-1976. [CrossRef] [PubMed]

4. Weinhold, N.; Ashby, C.; Rasche, L.; Chavan, S.S.; Stein, C.; Stephens, O.W.; Tytarenko, R.; Bauer, M.A.; Meissner, T.; Deshpande, S.; et al. Clonal selection and double-hit events involving tumor suppressor genes underlie relapse in myeloma. Blood 2016, 128, 1735-1744. [CrossRef] [PubMed]

5. Jimenez, C.; Jara-Acevedo, M.; Corchete, L.A.; Castillo, D.; Ordonez, G.R.; Sarasquete, M.E.; Puig, N.; Martinez-Lopez, J.; Prieto-Conde, M.I.; Garcia-Alvarez, M.; et al. A Next-Generation Sequencing Strategy for Evaluating the Most Common Genetic Abnormalities in Multiple Myeloma. J. Mol. Diagn. 2017, 19, 99-106. [CrossRef] [PubMed]

6. Rossi, A.; Voigtlaender, M.; Janjetovic, S.; Thiele, B.; Alawi, M.; Marz, M.; Brandt, A.; Hansen, T.; Radloff, J.; Schon, G.; et al. Mutational landscape reflects the biological continuum of plasma cell dyscrasias. Blood Cancer J. 2017, 7, e537. [CrossRef] [PubMed]

7. Moreaux, J.; Klein, B.; Bataille, R.; Descamps, G.; Maiga, S.; Hose, D.; Goldschmidt, H.; Jauch, A.; Reme, T.; Jourdan, M.; et al. A high-risk signature for patients with multiple myeloma established from the molecular classification of human myeloma cell lines. Haematologica 2011, 96, 574-582. [CrossRef] [PubMed]

8. Tessoulin, B.; Eveillard, M.; Lok, A.; Chiron, D.; Moreau, P.; Amiot, M.; Moreau-Aubry, A.; le Gouill, S.; Pellat-Deceunynck, C. p53 dysregulation in B-cell malignancies: More than a single gene in the pathway to hell. Blood Rev. 2017, 31, 251-259. [CrossRef] [PubMed]

9. Bykov, V.J.N.; Eriksson, S.E.; Bianchi, J.; Wiman, K.G. Targeting mutant p53 for efficient cancer therapy. Nat. Rev. Cancer 2017. [CrossRef] [PubMed]

10. Surget, S.; Descamps, G.; Brosseau, C.; Normant, V.; Maiga, S.; Gomez-Bougie, P.; Gouy-Colin, N.; Godon, C.; Bene, M.C.; Moreau, P.; et al. RITA (Reactivating p53 and Inducing Tumor Apoptosis) is efficient against TP53abnormal myeloma cells independently of the p53 pathway. BMC Cancer 2014, 14, 437. [CrossRef] [PubMed]

11. Tessoulin, B.; Descamps, G.; Moreau, P.; Maiga, S.; Lode, L.; Godon, C.; Marionneau-Lambot, S.; Oullier, T.; le Gouill, S.; Amiot, M.; et al. PRIMA-1Met induces myeloma cell death independent of p53 by impairing the GSH/ROS balance. Blood 2014, 124, 1626-1636. [CrossRef] [PubMed]

12. Soldevilla, B.; Millan, C.S.; Bonilla, F.; Dominguez, G. The TP73 complex network: Ready for clinical translation in cancer? Genes Chromosomes Cancer 2013, 52, 989-1006. [CrossRef] [PubMed]

13. Zawacka-Pankau, J.; Kostecka, A.; Sznarkowska, A.; Hedstrom, E.; Kawiak, A. p73 tumor suppressor protein: A close relative of p53 not only in structure but also in anti-cancer approach? Cell Cycle 2010, 9, 720-728. [CrossRef] [PubMed]

14. Kawano, S.; Miller, C.W.; Gombart, A.F.; Bartram, C.R.; Matsuo, Y.; Asou, H.; Sakashita, A.; Said, J.; Tatsumi, E.; Koeffler, H.P. Loss of $p 73$ gene expression in leukemias/lymphomas due to hypermethylation. Blood 1999, 94, 1113-1120. [PubMed]

15. Alexandrova, E.M.; Moll, U.M. Role of p53 family members p73 and p63 in human hematological malignancies. Leuk. Lymphoma 2012, 53, 2116-2129. [CrossRef] [PubMed]

16. Maas, A.M.; Bretz, A.C.; Mack, E.; Stiewe, T. Targeting p73 in cancer. Cancer Lett. 2013, 332, $229-236$. [CrossRef] [PubMed]

17. Teoh, P.J.; Bi, C.; Sintosebastian, C.; Tay, L.S.; Fonseca, R.; Chng, W.J. PRIMA-1 targets the vulnerability of multiple myeloma of deregulated protein homeostasis through the perturbation of ER stress via p73 demethylation. Oncotarget 2016, 7, 61806-61819. [CrossRef] [PubMed] 
18. Surget, S.; Chiron, D.; Gomez-Bougie, P.; Descamps, G.; Menoret, E.; Bataille, R.; Moreau, P.; le Gouill, S.; Amiot, M.; Pellat-Deceunynck, C. Cell death via DR5, but not DR4, is regulated by p53 in myeloma cells. Cancer Res. 2012, 72, 4562-4573. [CrossRef] [PubMed]

19. Engelmann, D.; Meier, C.; Alla, V.; Putzer, B.M. A balancing act: Orchestrating amino-truncated and full-length p73 variants as decisive factors in cancer progression. Oncogene 2015, 34, 4287-4299. [CrossRef] [PubMed]

20. Surget, S.; Lemieux-Blanchard, E.; Maiga, S.; Descamps, G.; le Gouill, S.; Moreau, P.; Amiot, M.; Pellat-Deceunynck, C. Bendamustine and melphalan kill myeloma cells similarly through reactive oxygen species production and activation of the p53 pathway and do not overcome resistance to each other. Leuk. Lymphoma 2014, 55, 2165-2173. [CrossRef] [PubMed]

21. Saha, M.N.; Jiang, H.; Yang, Y.; Reece, D.; Chang, H. PRIMA-1Met/APR-246 displays high antitumor activity in multiple myeloma by induction of p73 and Noxa. Mol. Cancer Ther. 2013, 12, 2331-2341. [CrossRef] [PubMed]

22. Moll, U.M.; Slade, N. p63 and p73: Roles in development and tumor formation. Mol. Cancer Res. 2004, 2, 371-386. [PubMed]

23. Polager, S.; Ginsberg, D. p53 and E2f: Partners in life and death. Nat. Rev. Cancer 2009, 9, 738-748. [CrossRef] [PubMed]

24. Conforti, F.; Sayan, A.E.; Sreekumar, R.; Sayan, B.S. Regulation of p73 activity by post-translational modifications. Cell Death Dis. 2012, 3, e285. [CrossRef] [PubMed]

25. Finzer, P.; Krueger, A.; Stohr, M.; Brenner, D.; Soto, U.; Kuntzen, C.; Krammer, P.H.; Rosl, F. HDAC inhibitors trigger apoptosis in HPV-positive cells by inducing the E2F-p73 pathway. Oncogene 2004, 23, 4807-4817. [CrossRef] [PubMed]

(C) 2017 by the authors. Licensee MDPI, Basel, Switzerland. This article is an open access article distributed under the terms and conditions of the Creative Commons Attribution (CC BY) license (http://creativecommons.org/licenses/by/4.0/). 